\title{
TRPM7 channels mediate the functional changes in cardiac fibroblasts induced by angiotensin II
}

\author{
SHA LI ${ }^{1-3}$, MINGJIANG LI ${ }^{1-3}$, XIN YI $^{1-3}$, FURONG GUO $^{1-3}$, YANLI ZHOU $^{1-3}$, SUQIN CHEN $^{1-3}$ and XIAN WU ${ }^{1-3}$ \\ ${ }^{1}$ Department of Cardiology, Renmin Hospital of Wuhan University; ${ }^{2}$ Cardiovascular Research Institute, \\ Wuhan University; ${ }^{3}$ Hubei Key Laboratory of Cardiology, Wuhan, Hubei 430060, P.R. China
}

Received October 11, 2016; Accepted March 20, 2017

DOI: $10.3892 /$ ijmm.2017.2943

\begin{abstract}
Transient receptor potential melastatin 7 (TRPM7), a bifunctional channel protein owning both cation permeability and kinase activity, plays an important role in the pathophysiological process of many cell types, such as vascular smooth muscle cells, human glioma cells and mouse cortical astrocytes. However, whether TRPM7 channels play a key role in the functional change of cardiac fibroblasts (CFs) induced by angiotensin II (Ang II) remains unknown. Using Cell Counting Kit-8 (CCK-8) assay, immunofluorescence assay, western blot analysis, RT-qPCR, RNA interference (RNAi) and whole-cell patch-clamp techniques, the present study aimed to explore the role of TRPM7 channels in the proliferation, differentiation and collagen synthesis of CFs induced by Ang II. Our data showed that Ang II time-dependently increased TRPM7 expression and TRPM7 currents in the CFs. Downregulation of TRPM7 attenuated the TRPM7 current density, and inhibited the proliferation, differentiation and collagen synthesis of CFs induced by Ang II. Our results identified the TRPM7 channel as a pivotal member associated with the functional change of CFs induced by Ang II, and suggest that the TRPM7 channel may represent a promising therapeutic strategy for the treatment of fibrosis-related cardiac diseases.
\end{abstract}

\section{Introduction}

Cardiac fibrosis, strongly associated with various cardiovascular pathological processes, leads to many cardiac diseases such as heart failure and types of arrhythmias especially atrial fibrillation as well as sudden cardiac death by causing ischemic and hypoxic changes in the myocardium, increasing myocardium stiffness, reducing systolic heart ejection and blocking electrical conduction (1). It is characterized by excessive production and deposition of extracellular matrix (ECM)

Correspondence to: Professor Mingjiang Li, Department of Cardiology, Renmin Hospital of Wuhan University, 238 Jiefang Road, Wuhan, Hubei 430060, P.R. China

E-mail: 754851539@qq.com

Key words: transient receptor potential melastatin 7, angiotensin II, cardiac fibroblasts, functional change proteins, including various types of collagen protein, which are mainly produced by cardiac fibroblasts (CFs) and contractile, hypersecretory, productive myofibroblasts derived from CFs suffering a myriad of pathological stimuli $(2,3)$. CFs are the most abundant cell type in the heart tissue, accounting for $\sim 75 \%$ of all cardiac cells and predominantly maintain the functional and structural integrity of the entire heart. For a long time, on account of their small size, comprising only $\sim 10-15 \%$ of the heart volume and having no excitability and contractibility, the pathological role of CFs was overlooked by most investigators. However, in recent years, research has shown that CFs play a vital role in the cardiac fibrogenesis cascade. Regardless of the initial stimuli, CFs are the final, decisive factor of the cardiac fibrosis process $(4,5)$. Therefore, restraining the activation and function of CFs has been identified as a promising therapeutic strategy for cardiac fibrosis-related diseases.

$\mathrm{Ca}^{2+}$ signaling is crucial for numerous cellular functions including gene expression, cell growth, proliferation, differentiation and death (6). Previous studies have shown that $\mathrm{Ca}^{2+}$ signaling is closely related to the initiation and development of fibrosis and is indispensable for the pro-fibrogenic effect of CFs (7-9). Runnels et al (10) identified a new type of $\mathrm{Ca}^{2+}$-permeable ion channel named transient receptor potential melastatin 7 (TRPM7) in mammals. Our team and other investigators have elucidated that TRPM7 channels are highly expressed on the membrane of CFs, and may be the only calcium permeable cation channel on the CF membrane, bearing the $\mathrm{Ca}^{2+}$-influx signal and closely associated with the cardiac fibrosis process $(4,11)$. However, whether TRPM7 channels contribute to cardiac fibrogenesis by regulating functional changes of $\mathrm{CF}$ s remains elusive. Therefore, by stimulating CFs with angiotensin II (Ang II) in vitro, we demonstrated that TRPM7 channels modulate the proliferation and differentiation capacity of CFs by mediating $\mathrm{Ca}^{2+}$ signaling on the membrane, and then increase collagen synthesis finally resulting in myocardial interstitial fibrosis.

\section{Materials and methods}

Animals, CFs and reagents. Sprague-Dawley (SD) rats with an SPF status (1-3 days old) were purchased from the Animal Experimental Center of Wuhan University and the Center for Disease Control and Prevention of Hubei Province. Dulbecco's modified Eagle's medium (DMEM)-F12 was purchased from HyClone (Logan, UT, USA). Phosphate-buffered saline (PBS), 
penicillin/streptomycin and trypsin were purchased from Jinuo Co. (Hangzhou, China). Fetal bovine serum (FBS) was obtained from Invitrogen and Gibco Life Technologies (Carlsbad, CA, USA). The sources of the various primary and secondary antibodies are provided in the text. Ang II (A9525) and collagenase type II were acquired both from Sigma-Aldrich (St. Louis, MO, USA). CFs were divided into five groups: i) blank control (BC), CFs with no intervention; ii) negative control (NC), CFs transfected with adenoviral vectors carrying green fluorescent protein (GFP) only without the TRPM7-small interfering RNA (siRNA) sequence; iii) $\mathrm{NC}+$ Ang II, CFs transfected with adenoviral vectors carrying GFP only without the TRPM7-siRNA sequence and then treated with $1 \mu \mathrm{M} / 1$ Ang II for $24 \mathrm{~h}$; iv) TRPM7-siRNA, CFs transfected with adenoviral vectors carrying GFP and the TRPM7-siRNA sequence; v) TRPM7-siRNA+Ang II, CFs transfected with adenoviral vectors carrying GFP and the TRPM7-siRNA sequence and then treated with $1 \mu \mathrm{M} / 1$ Ang II for $24 \mathrm{~h}$.

Primary $C F$ isolation and culture. Our experimental protocol concerning the acquisition and use of animal heart tissues was approved by the Ethics Committee of Renmin Hospital of Wuhan University. Primary CFs were obtained from 1- to 3-day-old SD rat pups applying the method of enzymatic digestion and differential attachment as described in our previous study with a few adjustments (4). Briefly, shredded heart tissues were first enzymatically digested with $0.125 \%$ trypsin for $10 \mathrm{~min}$ and the supernatant was discarding. Then, $0.125 \%$ trypsin was supplemented with $0.08 \%$ collagenase type II for 5 min 8 times and the supernatant was collected. After centrifugation, the cells were resuspended in DMEM-F12 (HyClone) containing 10\% FBS (Gibco) and $1 \%$ penicillin/streptomycin for differential attachment. After $1.5 \mathrm{~h}$, the cell culture solution was completely replaced with fresh culture medium and the dish was placed in a constant temperature incubator at $37^{\circ} \mathrm{C}$ with $5 \% \mathrm{CO}_{2}$. Every 2-3 days, the cells were washed with PBS for 3 times and the complete medium was exchanged. When the cell density reached $90 \%$, passaging was performed by digesting the cells using $0.25 \%$ trypsin plus $0.5 \mathrm{mM}$ EDTA. In general, the cells were passaged to 3-5 generations for subsequent experimental use.

Western blot analysis. Western blot analysis was carried out to assess the protein expression levels of TRPM7, $\alpha$-smooth muscle actin ( $\alpha$-SMA), Ki-67, PCNA, collagen I and III in the CFs following the different treatments. The cell total protein was extracted by cracking CFs in RIPA lysis buffer (AS1004; Aspen Biological, Wuhan, China) including proteinase inhibitor cocktails. Protein concentrations were probed with the BCA protein assay kit (AS1086; Aspen Biological) for equalizing the protein content for each group. Equivalent amounts of proteins $(40 \mu \mathrm{g} / \mathrm{lane})$ were separated by using distinct sodium dodecyl sulfate-polyacrylamide gel electrophoresis (SDS-PAGE) (10\% for GAPDH, $\alpha$-SMA and Ki-67; $8 \%$ for TRPM7, collagen I and III; 12\% for PCNA). Subsequently, the separated proteins were transferred onto PVDF membranes, blocked with 5\% skim milk, and dissolved in TBST for $1 \mathrm{~h}$ at room temperature (RT). The blots were respectively incubated with various primary antibodies against $\alpha$-SMA (TDY210,
1:5,000), Ki-67 (bs-2130R, 1:500), PCNA (ab92552, 1:1,500), collagen I (ab6308, 1:1,000), collagen III (ab7778, 1:500), TRPM7 (ab109438, 1:1,000) and GAPDH (ab37168, 1:10,000) (all from Abcam, Cambridge, MA, USA) at $4^{\circ} \mathrm{C}$ overnight. After being washed with TBST for 3 times ( 5 min each time), the membranes were treated with either HRP-conjugated goat anti-rabbit $(074-1506,1: 10,000)$ or goat anti-mouse (074-1806, 1:10,000) (both from Kirkegaard and Perry Laboratories, Inc., Gaithersburg, MD, USA) secondary antibodies for $1 \mathrm{~h}$ at RT. The chemiluminescence of the blots was determined with an ECL kit (AS1059; Aspen Biological), and AlphaEaseFC software (Alpha Innotech, San Leandro, CA, USA) was employed to analyze the optical density. GAPDH was used as the internal control.

$R T-q P C R$. Total RNA of the CFs grown on 6-well plates was extracted with TRIzol reagent (15596-026; Invitrogen $^{\mathrm{TM}}$ ) and reverse transcribed to cDNA according to the manufacturer's instructions of Toyobo First Strand cDNA synthesis kit (ReverTra Ace- $\alpha$-, FSK-100; Toyobo, Osaka, Japan). Quantitative PCR was carried out in three stages: first, $95^{\circ} \mathrm{C}$ for $1 \mathrm{~min}$ for pre-denaturation; second, a 40 -cycle reaction at $95^{\circ} \mathrm{C}$ for $15 \mathrm{sec}, 58^{\circ} \mathrm{C}$ for $20 \mathrm{sec}$ and $72^{\circ} \mathrm{C}$ for $45 \mathrm{sec}$; third, assessing the temperature from $60^{\circ} \mathrm{C}$ to $95^{\circ} \mathrm{C}\left(1^{\circ} \mathrm{C}\right.$ every $\left.20 \mathrm{sec}\right)$ for obtaining the melting curve using SYBR ${ }^{\oplus}$ Premix Ex Taq ${ }^{\mathrm{TM}}$ kit (RR420A; Takara, Otsu, Japan) on StepOne ${ }^{\mathrm{TM}}$ Real-Time PCR apparatus (Life Technologies, Carlsbad, CA, USA). All the primers used in this study were designed by Invitrogen Biotechnology Co., Ltd. (Shanghai, China) and are as follows: TRPM7, 5'-GTA CCTGGTCAGAGCACG ATGT-3' and 5'-TGGTATGGA TTTGGGTTTCATC-3'; collagen I, 5'-AACTGGTACATC AGCCCAAACC-3' and 5'-ATCGGAACCTTCGCTTC CAT-3'; collagen III, 5'-ATGT GTCTGCGACTCGGGAT-3' and 5'-ACAGGAGCAGGTGTAGAAGGC-3'; GAPDH, 5'-CGCTAACATCAAATGGGGTG-3' and 5'-TTGCTG ACAATCTTGAGGGAG-3'; actin, 5'-CGTTGACATCCG TAAAGACCTC-3' and 5'-TAGGAGCCAGGGCAGTAA TCT-3'. GADPH and actin gene were employed as the internal control.

$R N A$ interference. Adenovirus vectors carrying small interfering RNA (siRNA) targeting rat TRPM7 and green fluorescent protein gene (PHBAd-U6-Scramble-CMV-GFP) were designed and synthesized by Hanheng Biological Co. (Shanghai, China). The silencing oligonucleotide sequences are as follows: siRNA1, 5'-GATGTCAGATTTGTCAGCA ACTTGT-3'; siRNA2,5'-GCTCAGAATCTTATTGATGAT-3'. In addition, the adenovirus vectors with GFP only, without the knockdown sequence were applied as the negative control group (NC). According to the preliminary experimental data, we defined the multiplicity of infection of the adenovirus infecting $\mathrm{CFs}$ as $50(\mathrm{MOI}=50)$. $\mathrm{CFs}$ inoculated in 6-well plates were treated with the viruses for $2 \mathrm{~h}$ with DMEM-free/F12. After $24 \mathrm{~h}$, the infected cells were examined by fluorescence microscopy. After $72 \mathrm{~h}, \mathrm{RT}$-qPCR and western blot analysis were conducted to monitor the silencing efficiency of TRPM7 at the mRNA and protein levels, respectively.

Recording of the TRPM7 currents. The whole-cell patch-clamp experiments were performed as previously reported $(4,12)$ for 
recording the TRPM7 currents on the CF membrane under various conditions. The internal solution perfused in the glass electrode contained: $145 \mathrm{mmol} / \mathrm{l} \mathrm{Cs}-\mathrm{mesilate}, 8 \mathrm{mmol} / \mathrm{l} \mathrm{NaCl}$, $10 \mathrm{mmol} / \mathrm{l}$ EGTA and $10 \mathrm{mmol} / \mathrm{l} \mathrm{HEPES}, \mathrm{PH}$ value adjusted to 7.2 with $\mathrm{CsOH}$. The standard extracellular Tyrode solution contained: $140 \mathrm{mmol} / \mathrm{l} \mathrm{NaCl}, 5 \mathrm{mmol} / 1 \mathrm{KCl}, 2 \mathrm{mmol} / 1 \mathrm{CaCl}_{2}$, $20 \mathrm{mmol} / \mathrm{l} \mathrm{HEPES}, 10 \mathrm{mmol} / 1$ glucose adjusting $\mathrm{PH}$ value to 7.4 with $\mathrm{NaOH}$. Borosilicate glass electrodes were pulled using a microelectrode puller (PB-7; Narishige, Tokyo, Japan) holding the resistance at 3-5 M $\Omega$ when filled with pipette solution. The whole cell configuration was set up so the impedance was rapidly increased and a slight negative pressure was applied leading to the rupture of the membranes. For acquiring the current-voltage relationships (I-V curves), voltage step protocol was applied with a stimulus voltage ranging from -120 to $+100 \mathrm{mV}$ lasting for $500 \mathrm{msec}$. TRPM7 currents were recorded by utilizing an Axopatch 200B Amplifier (Molecular Devices, LLC, Sunnyvale, CA, USA) and Pulse+Pulsefit 8.8 software program (HEKA Elektronik, Lambrecht, Germany). All the data were digitized at $5 \mathrm{kHz}$, filtered at $2 \mathrm{kHz}$ and normalized according to cell size (Cslows) as $\mathrm{pA} / \mathrm{pF}$.

Cell Counting Kit-8(CCK-8) assay. CCK-8 assay was applied to assess the activity and proliferation ability of the CFs. Cells were inoculated in 96-well culture plates at a density of $2 \times 10^{4}$ cells $/ \mathrm{ml}$ (200 $\mu 1, \sim 4,000$ cells/well). After treatment with the viruses or Ang II for $24 \mathrm{~h}$, cells in each well were washed with PBS for 2 times and fresh complete medium containing $20 \mu \mathrm{l} \mathrm{CCK}-8$ reagent was added. The plates then were incubated in a constant temperature incubator with $5 \% \mathrm{CO}_{2}$ at $37^{\circ} \mathrm{C}$ for $4 \mathrm{~h}$. Finally, the absorbance at $450 \mathrm{~nm}$ was measured with a microplate reader (Tecan infinite M200; Tecan, Durham, NC, USA).

Immunofluorescence assay. CFs growing on rinsed aseptic coverslips in 6-well plate were washed with PBS for 2 times, fixed with $4 \%$ paraformaldehyde for $30 \mathrm{~min}$, permeabilized with $0.1 \%$ Triton X-100 for 10 min and then blocked with 5\% BSA for $1 \mathrm{~h}$. After incubation with the primary antibody against $\alpha$-SMA (ab3280; Abcam) overnight at $4^{\circ} \mathrm{C}$, the cells were then incubated with a red fluorescence-marked secondary antibody (AS1109; Aspen Biological) for $1 \mathrm{~h}$ at RT. The coverslips were mounted on glass slides with fluoromount-G resistance to fluorescence quenching containing 4',6-diamidino-2-phenylindole (DAPI) (AS105; Aspen Biological). The images were observed and saved using fluorescence microscopy with 200X objective.

Statistical analysis. SPSS 17.0 (SPSS, Inc., Chicago, IL, USA) was used for data analysis. All data are presented as mean \pm SD. Differences among groups were assessed with one-way analysis of variance (ANOVA) followed by least significant difference-t (LSD- $t$ ) test and statistically significant differences were defined as having P-values $<0.05$.

\section{Results}

Ang II induces time-dependent TRPM7 expression in CFs. To determine the optimal time-point of $1 \mu \mathrm{M} / 1$ Ang II for generating cardiac fibrosis, western blot technology was applied to measure the TRPM7 channel protein expression levels in Ang II-intervened primary cultured CFs at five different time-
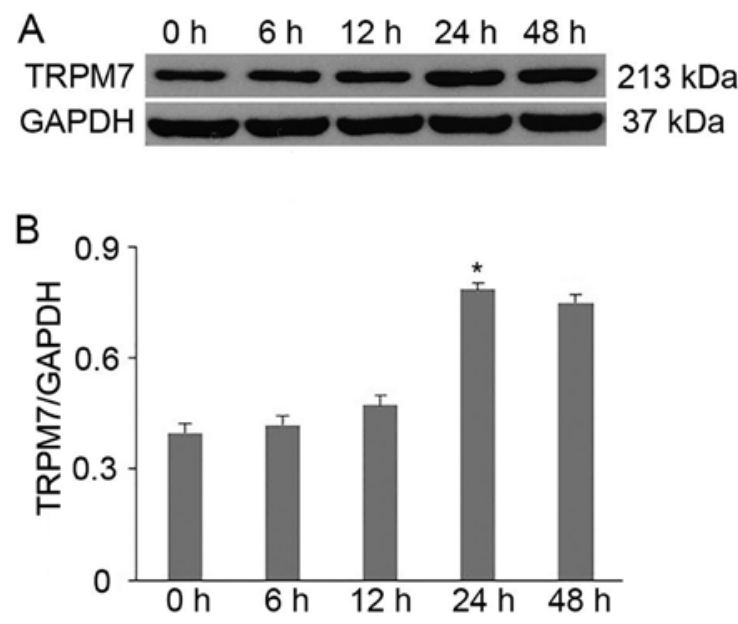

Figure 1. Angiotensin II (Ang II) induces time-dependent transient receptor potential melastatin 7 (TRPM7) expression in cardiac fibroblasts (CFs). (A) Western blot showing TRPM7 expression at five different time-points $(0,6,12,24$ and $48 \mathrm{~h})$ after Ang II treatment $(1 \mu \mathrm{M})$. GAPDH served as an internal control. (B) Data analysis of the western blotting indicated that TRPM7 expression reached the highest level at $24 \mathrm{~h}$ compared with the other time-points ( ${ }^{*} \mathrm{p}<0.01$ vs. 0,6 and $\left.12 \mathrm{~h}\right)$ and a slight decrease was noted at $48 \mathrm{~h}$, but with no statistical significance ( $>0.05,48$ vs. $24 \mathrm{~h}$ ).

points $(0,6,12,24$ and $48 \mathrm{~h})$. As shown in Fig. 1, our data indicated that TRPM7 channels were expressed in the primary cultured CFs $(0 \mathrm{~h})$. In addition, the TRPM7 channel protein was time-dependently increased after Ang II intervention and reached the highest level at $24 \mathrm{~h}$ compared with the other timepoints ( $\mathrm{p}<0.01$ vs. 0,6 and $12 \mathrm{~h}$ ) (Fig. 1B); a slight decrease was noted at $48 \mathrm{~h}$, but with no statistical significance ( $>0.05$, 48 vs. 24 h) (Fig. 1B). Therefore, we selected treatment with Ang II for $24 \mathrm{~h}$ as the optimum time-point for the rest of our experiments.

Gene silencing efficiency of TRPM7 in CFs. In order to knockdown TRPM7 channel protein in CFs, we used an adenovirus vector carrying an siRNA targeting the rat TRPM7 gene (Ad-TRPM7-siRNA) as described in Materials and methods. As revealed in Fig. 2B and C, TRPM7 expression in the BC and $\mathrm{NC}$ groups had no statistical difference ( $\mathrm{p}>0.05, \mathrm{NC}$ vs. BC) indicating that the adenovirus vector carrying GFP only did not affect the expression of TRPM7 channels in the CFs. TRPM7-siRNA1 and TRPM7-siRNA2 obviously reduced the protein expression level of the TRPM7 channel by $63.5 \pm 2.3$ and $84.5 \pm 4.2 \%$, respectively compared to the NC group $(\mathrm{p}<0.01$ vs. NC) (Fig. 2B). The results of RT-qPCR were consistent with the western blot analysis indicating that TRPM7-siRNA1 and TRPM7-siRNA2 significantly decreased the mRNA level of TRPM7 in the CFs by $45.6 \pm 6.0$ and $77.2 \pm 4.8 \%$ respectively $(\mathrm{p}<0.01$ vs. NC) (Fig. 2C). Hence, TRPM7-siRNA2 was selected for our subsequent gene silencing experiments due to its higher efficiency ( $\mathrm{p}<0.05$ vs. TRPM7-siRNA1; Fig. 2B) (p<0.01 vs. TRPM7-siRNA1; Fig. 2C).

Silencing of TRPM7 attenuates Ang II-induced elevation in TRPM7 currents in CFs. To further demonstrate the expression of TRPM7 channels increased by Ang II in CFs, we performed whole-cell patch-clamp technique using a voltage step protocol (the stimulus voltage ranging from -120 to $+100 \mathrm{mV}$ ) to detect 

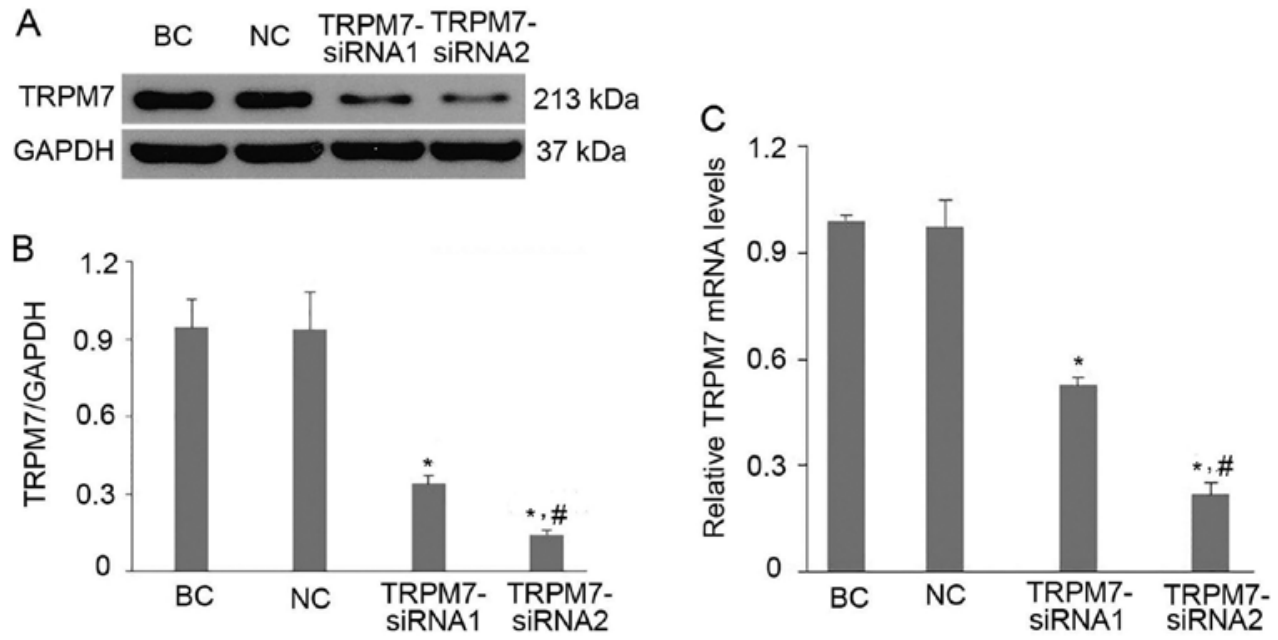

Figure 2. Gene silencing efficiency of transient receptor potential melastatin 7 (TRPM7) in cardiac fibroblasts (CFs). (A and B) Western blotting exhibited that CFs transfected with adenoviral vectors carrying specific TRPM7-siRNA expressed less TRPM7 protein. (B) Quantification of the western blotting. GAPDH served as an internal control. "p<0.01 vs. NC; ${ }^{*} \mathrm{p}<0.05$ vs. TRPM7-siRNA1. (C) RT-PCR displayed the same trend changes. Actin served as the internal control " $\mathrm{p}<0.01$ vs. NC; ${ }^{*} \mathrm{p}<0.01$ vs. TRPM7-siRNA1. Quantative analysis of TRPM7 protein and mRNA level identified TRPM7-siRNA2 as the optimal sequence due to its higher efficiency. BC, blank control (CFs with no intervention); NC, negative control (CFs transfected with adenoviral vectors carrying green fluorescent protein (GFP) only without TRPM7-siRNA sequence).
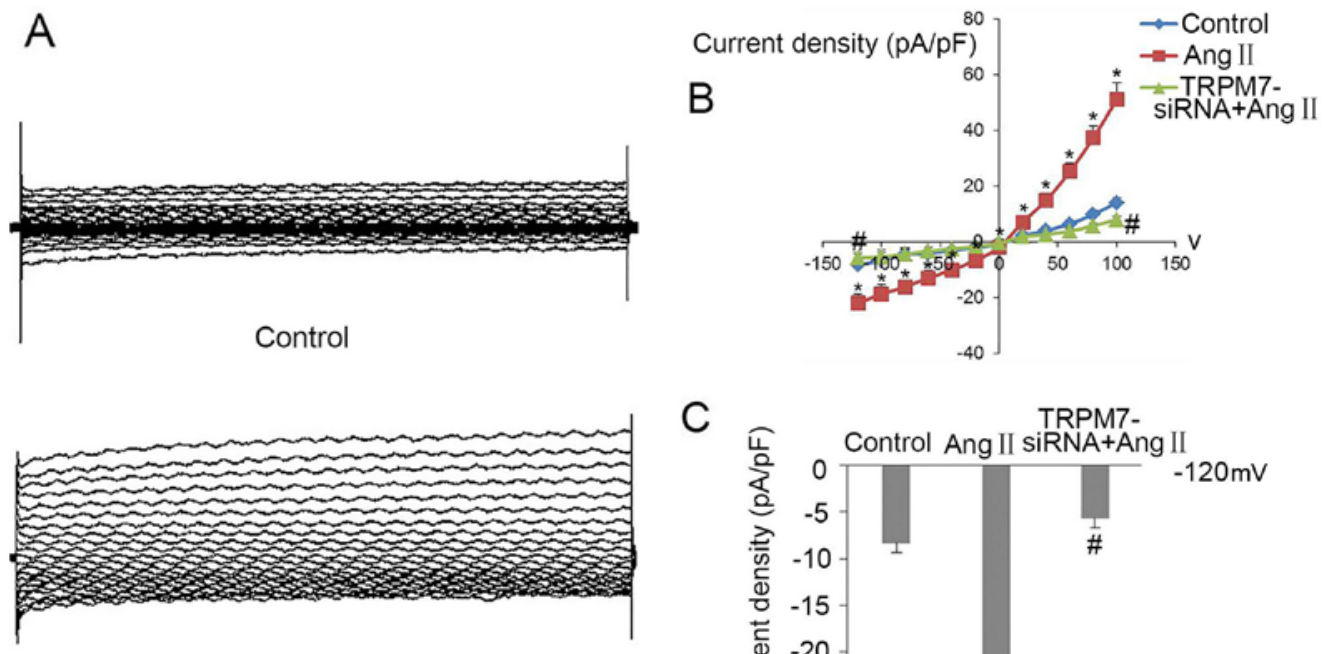

C
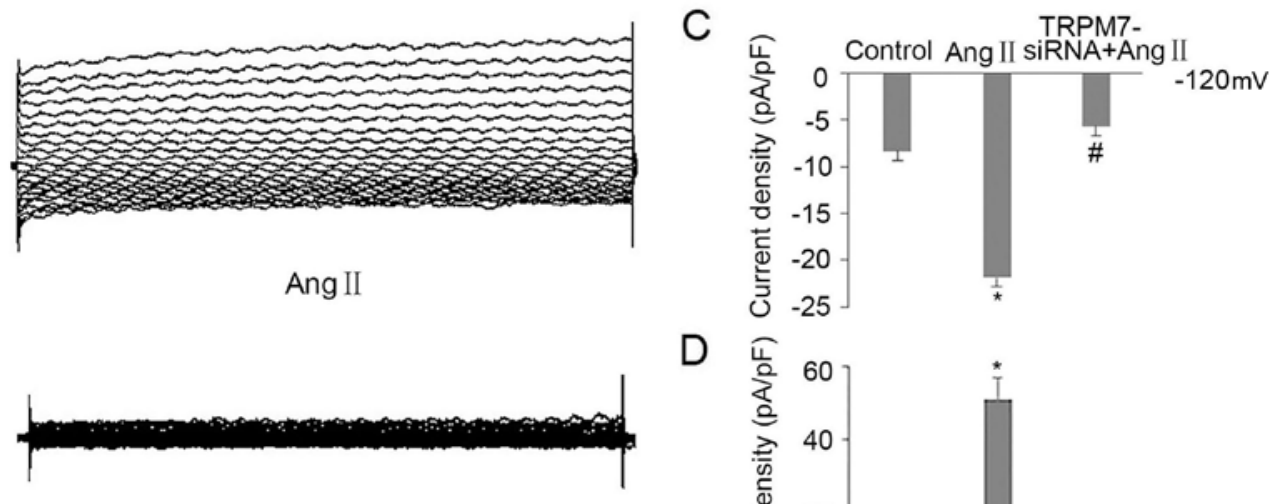

D

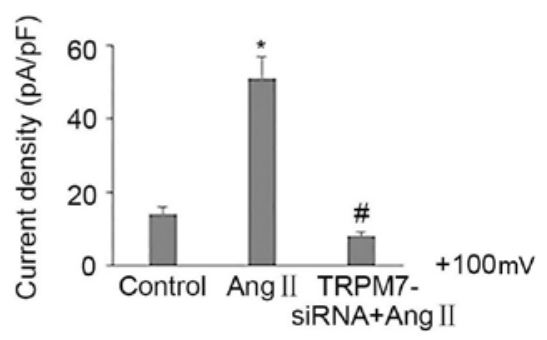

Figure 3. Silencing of transient receptor potential melastatin 7 (TRPM7) attenuates angiotensin II (Ang II)-induced elevation of TRPM7 currents in cardiac fibroblasts (CFs). TRPM7 currents were assessed using whole-cell path clamp technique with the stimulus voltage ranging from -120 to $+100 \mathrm{mV}$. (A) Present graphs recorded in the CF membrane under basal condition, treated with Ang II and Ad-TRPM7-siRNA+Ang II status. (B) Representative I-V curves. Ang II markedly amplified the TRPM7 current density ( $\mathrm{n}=6$; " $\mathrm{p}<0.05 \mathrm{vs}$. control), while TRPM7-siRNA significantly reduced Ang II-induced current density ( $\mathrm{n}=6$; ${ }^{\#} \mathrm{p}<0.05$ vs. Ang II). (C and D) Quantative analysis of current density values at -120 and $+100 \mathrm{mV}$ ( $\mathrm{n}=6$; ${ }^{*} \mathrm{p}<0.05$ vs. control; $\mathrm{n}=6$; ${ }^{*} \mathrm{p}<0.05$ vs. Ang II).

the TRPM7 current amplitude on the CF membrane under basal condition, following treatment with Ang II and Ad-TRPM7siRNA. As shown in Fig. 3A, an inward and outward rectifying current was recorded immediately in $\mathrm{CFs}$ after the whole-cell configuration was established. At $-120 \mathrm{mV}$, the current was inward-rectifying, its amplitude gradually weakened and turned into an outward-rectifying current at $0 \mathrm{mV}$ around, then was gradually elevated and reached the highest level at $+100 \mathrm{mV}$, in $\sim 500 \mathrm{msec}$. In addition, the current-voltage relationships (I-V curves) (Fig. 3B) showed that the inward and 

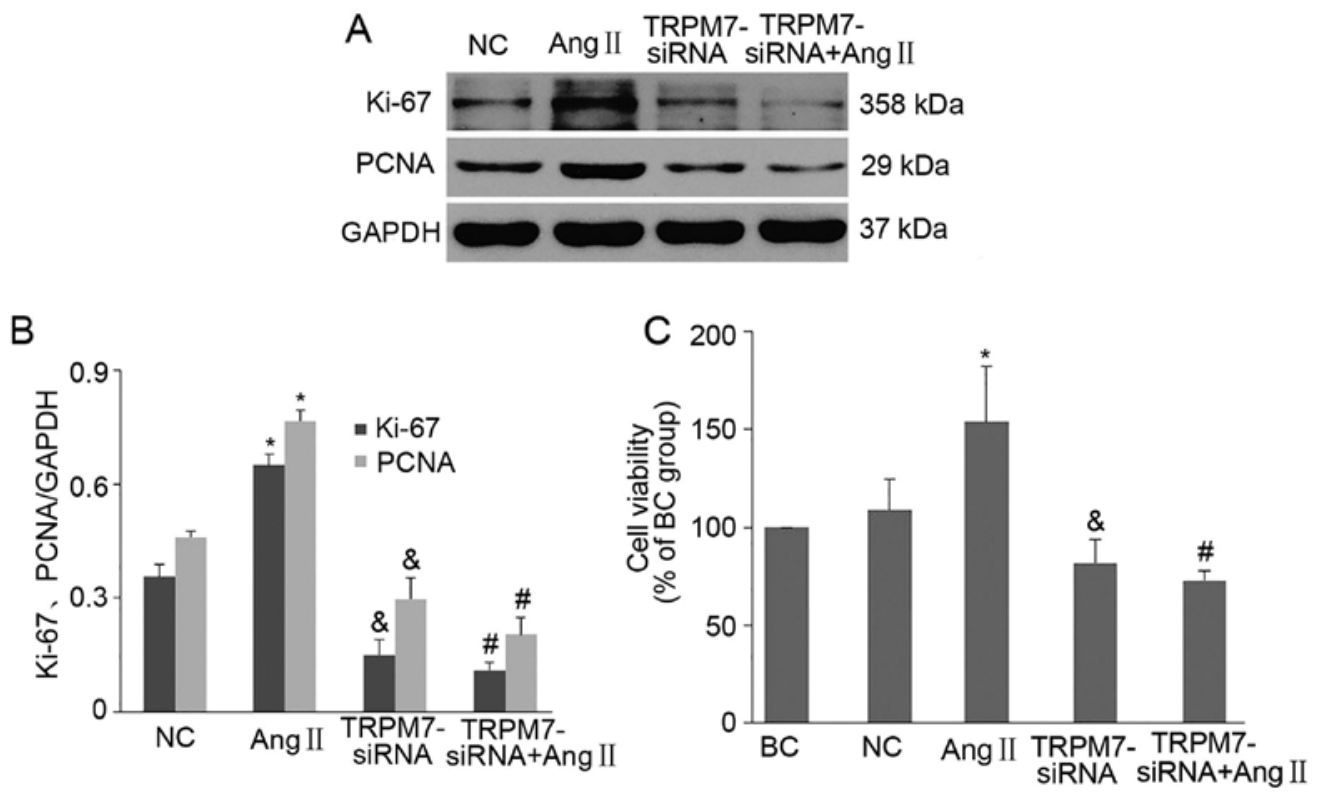

Figure 4. Knockdown of transient receptor potential melastatin 7 (TRPM7) inhibits the proliferation induced by angiotensin II (Ang II) in cardiac fibroblasts (CFs). (A and B) Western blotting showed that Ang II markedly increased Ki-67 and PCNA protein expression levels which represent cell proliferation ability ("p $<0.05$ vs. NC), while TRPM7-siRNA abrogated the Ang II effect ("p<0.05 vs. Ang II). (C) CCK-8 assay showed the same trends ("p< $<0.05$ vs. NC; ${ }^{\#} \mathrm{p}<0.05$ vs. Ang II). TRPM7-siRNA inhibited Ang II-elicited CF proliferation $\left({ }^{\#} \mathrm{p}<0.01\right.$ vs. Ang II), as well as the proliferation of CFs in basal condition $\left({ }^{\circledR} \mathrm{p}<0.05\right.$ vs. NC).

outward rectifying current density of TRPM7 in the CFs was small in the basal condition, but dramatically increased after CFs were treated with Ang II for $24 \mathrm{~h}$ (the inward current density was $\sim 2$-fold higher compared to the control, the outward current density was $\sim 3$-fold higher compared to control, $\mathrm{n}=6$, $\mathrm{p}<0.05$ vs. control) then impaired when Ang II-induced CFs were intervened with Ad-TRPM7-siRNA (the inward current density was $\sim 75 \%$ impaired compared with that of Ang II, the outward current density was $\sim 90 \%$ impaired than that of Ang II, $n=6, p<0.05$ vs. Ang II). The quantative current density values at -120 and $+100 \mathrm{mV}$ exhibited the same change trends among the three groups $(n=6, p<0.01$ vs. control; $n=6, p<0.01$ vs. Ang II) (Fig. 3C and D). These electrophysiological results combined with the result of the above experiments illustrated that Ang II increased and Ad-TRPM7-siRNA decreased Ang II-induced expression of TRPM7 and TRPM7 currents in the CFs.

Knockdown of TRPM7 inhibits the proliferation induced by Ang II in CFs. It was reported that TRPM7 channels influence the growth and proliferation of many cell types (13). Here, we aimed to ascertain for the first time whether TRPM7 channels play a critical role in the proliferation of CFs. Firstly, we used CCK-8 assay to detect the proliferation abilities of CFs under various conditions. We found that pretreatment of CFs with $1 \mu \mathrm{M} / 1$ Ang II for $24 \mathrm{~h}$ significantly increased the proliferative ability of CFs ( $p<0.05$ vs. NC) (Fig. 4C). Downregulation of TRPM7 in CFs with Ad-TRPM7-siRNA markedly reduced Ang II-induced $\mathrm{CF}$ proliferation ( $\mathrm{p}<0.05 \mathrm{vs}$. Ang II) (Fig. 4C). To further confirm the role of TRPM7 channels in CF proliferation, cell cyclerelated regulatory protein $\mathrm{Ki}-67$ and proliferating cell nuclear antigen (PCNA) were employed to assess the CF proliferative rate. Fig. 4A and $\mathrm{B}$ shows that Ang II intervention prominently promoted $\mathrm{Ki}-67$ and PCNA protein expression and treatment with Ad-TRPM7-siRNA notably inhibited Ang II-elicited protein expression in the CFs $(\mathrm{p}<0.01$ vs. $\mathrm{NC} ; \mathrm{p}<0.01$ vs. Ang II) (Fig. 4B). In addition, we found that TRPM7-siRNA not only inhibited Ang II-elicited CF proliferation ( $p<0.01$ vs. Ang II) (Fig. 4B and C) but also suppressed the proliferation of CFs in basal condition ( $\mathrm{p}<0.05$ vs. NC) (Fig. 4B and C). Together, these results indicated that TRPM7 channels are involved in the proliferation of CFs, whereas knockdown of TRPM7 by siRNA inhibited the proliferation induced by Ang II.

Downregulation of TRPM7 suppresses the differentiation of CFs induced by Ang II. The pathophysiological process of CF differentiation into hypersecretory, excessive ECM-formating myofibroblasts plays a key role during cardiac fibrosis (3). Therefore, we decided to perform immunofluorescence assay and western blot technique to detect the expression of $\alpha$-SMA indirectly reflecting the differentiation ability of CFs. As shown in Fig. 5A, CFs passaged for 3-5 generations expressed a low level of $\alpha$-SMA while Ang II treatment obviously increased the expression of $\alpha$-SMA which was reduced in CFs pretreated with Ad-TRPM7-siRNA. The variation trends of $\alpha$-SMA protein acquired from western blot technology were consistent with the immunofluorescence assay $(\mathrm{p}<0.05$ vs. NC; p<0.05 vs. Ang II) (Fig. 5C). Furthermore, we found that TRPM7-siRNA inhibited Ang II-elicited CF differentiation ( $p<0.05$ vs. Ang II) (Fig. 5C) as well as the differentiation of CFs in basal condition ( $\mathrm{p}<0.05$ vs. NC) (Fig. 5C). These data clarified that TRPM7 channels were also associated with basal and Ang II-caused CF differentiation effect while silencing TRPM7 observably weakened the differentiation ability of the CFs.

Inhibitory effect of TRPM7-silencing on collagen synthesis of CFs induced by Ang II. In the pathological process of cardiac 
A
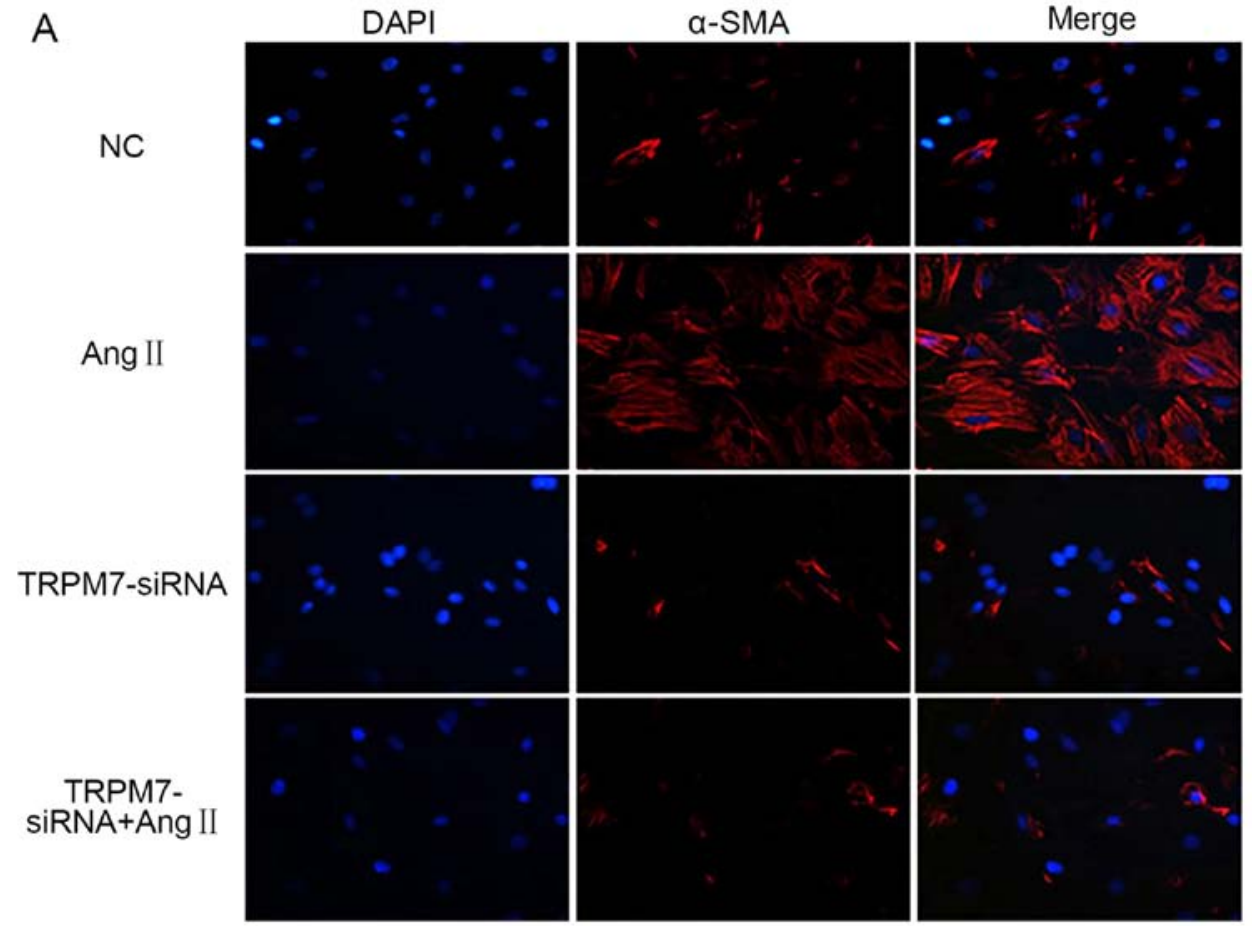

B
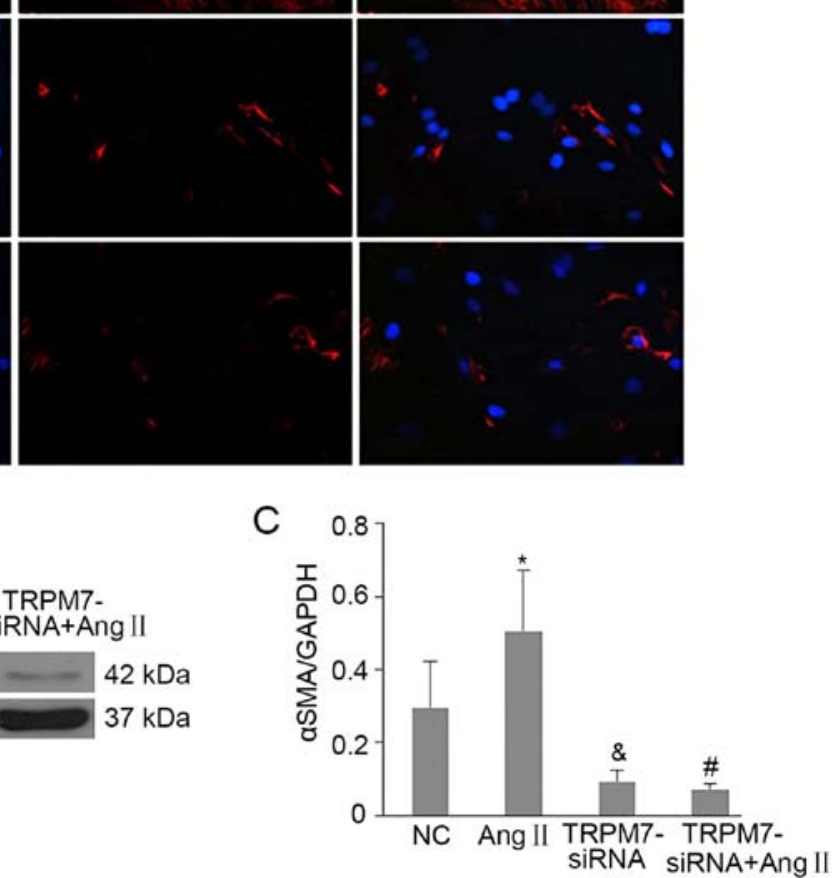

Figure 5. Downregulation of transient receptor potential melastatin 7 (TRPM7) suppresses the differentiation of cardiac fibroblasts (CFs) induced by angiotensin II (Ang II). (A) Immunofluorescence images show the significant suppression of Ang II-induced CF differentiation by downregulation of TRPM7. CF cell nuclei were stained with DAPI (blue). (B and C) Western blot analysis was used to assess $\alpha$-smooth muscle actin ( $\alpha$-SMA) expression levels among the different groups ( ${ }^{*} \mathrm{p}<0.05$ vs. NC; ${ }^{\#} \mathrm{p}<0.05$ vs. Ang II). Furthermore, TRPM7-siRNA inhibited Ang II-elicited CF differentiation $\left({ }^{\# p}<0.05\right.$ vs. Ang II) as well as the differentiation of CFs in basal condition $\left({ }^{\&} \mathrm{p}<0.05 \mathrm{vs}\right.$. NC).

fibrosis, ECM proteins are considerably produced and excessively deposited in normal heart tissues when the heart responds to various pathological stimuli such as pressure overload or acute myocardium infarction. Collagen I and III are two major components of ECM proteins, thus they were chosen as fibrosis biomarkers in the present study. Coincubation with Ang II for $24 \mathrm{~h}$ significantly increased the expression levels of collagen I and III in the CFs which were markedly lowered by Ad-TRPM7siRNA ( $p<0.05$ vs. NC; $p<0.01$ vs. NC+Ang II) (Fig. 6A and B). Quantification of the western blot data found that Ang II increased collagen I and III levels by $207 \pm 108$ and $48.9 \pm 9.8 \%$, respectively ( $<<0.05$ vs. NC) (Fig. 6B) and TRPM7-siRNA reduced Ang II-induced collagen I and III synthesis by $85.0 \pm 5.3$ and $71.6 \pm 9.6 \%$, respectively ( $<<0.05$ vs. NC+Ang II) (Fig. 6B). Quantification of the RT-qPCR data revealed a similar tend: Ang II increased collagen I and III mRNA levels by $80.9 \pm 26.3$ and $44.6 \pm 20.9 \%$, respectively ( $\mathrm{p}<0.05$ vs. NC) (Fig. 6C) while transfection of CFs with Ad-TRPM7-siRNA reduced AngII-elicited collagen I and III mRNA levels by $69.9 \pm 7.2$ and $60.3 \pm 11.2 \%$, respectively ( $<<0.05$ vs. NC+Ang II) (Fig. 6C). We also found that TRPM7-siRNA inhibited Ang II-elicited CF collagen synthesis ( $<<0.05$ vs. NC+Ang II) (Fig. $6 \mathrm{~B}$ and $\mathrm{C}$ ) as well as the collagen synthesis of CFs in basal condition ( $p<0.05$ vs. NC) (Fig. 6B and C). Taken together, the findings indicated that Ang II evoked an increase in collagen synthesis by CFs resulting in cardiac fibrosis while inhibition of TRPM7 expression prohibited the pro-fibrogenic effect of Ang II.

\section{Discussion}

The transient receptor potential (TRP) channel superfamily is a series of unique cation channels (14), firstly cloned in Drosophila (15), containing 28 TRP channels classified into six subfamilies: TRPC, TRPV, TRPM, TRPA, TRPML and TRPP. They are expressed in various tissues and involved in manifold basal cellular functions such as $\mathrm{Ca}^{2+}$ signaling, cell contraction, proliferation and death (16). Recently, the melastatin subfamily, member seven (TRPM7) ion channel has attracted great attention due to its distinctive structure that possesses a fusion which couples a functional C-terminal $\alpha$-type serine/threonine protein kinase region to a cation channel (17). The channel domain and the kinase region are responsible for regulating transmembrane 


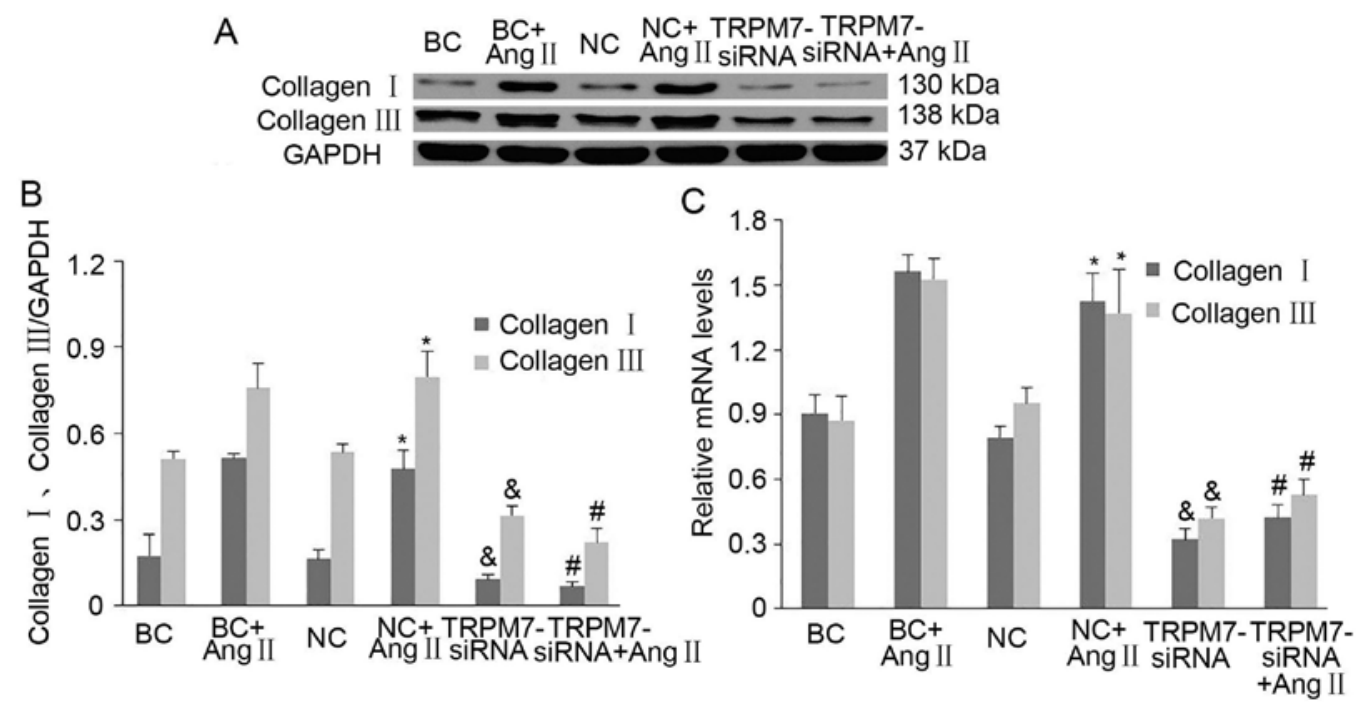

Figure 6. Inhibitory effect of transient receptor potential melastatin 7 (TRPM7) silencing on collagen synthesis of cardiac fibroblasts (CFs) induced by angiotensin II (Ang II). (A) Representative bands show the collagen I and III formation in CFs with different treatments. (B) Quantification of collagen synthesis of CFs among the various groups. Ang II significantly increased collagen I and III expression level by $207 \pm 108$ and $48.9 \pm 9.8 \%$, respectively ( $\mathrm{p}<0.05$ vs. NC) while transfection of CFs with Ad-TRPM7-siRNA reduced Ang II-elicited collagen I and III expression increase by $85.0 \pm 5.3$ and $71.6 \pm 9.6 \%$, respectively ( ${ }^{\#} \mathrm{p}<0.05$ vs. NC+Ang II). (C) RT-PCR showed that Ang II increased collagen I and III mRNA levels by $80.9 \pm 26.3$ and $44.6 \pm 20.9 \%$, respectively ( $" \mathrm{p}<0.05$ vs. NC), while transfection of CFs with Ad-TRPM7-siRNA reduced Ang II-elicited collagen I and III mRNA level by $69.9 \pm 7.2$ and $60.3 \pm 11.2 \%$, respectively $(\# p<0.05$ vs. NC+Ang II). In addition, TRPM7-siRNA inhibited Ang II-elicited CF collagen synthesis $(\# p<0.05$ vs. NC+Ang II), as well as the collagen synthesis of CFs in a basal condition $\left({ }^{\circledR} \mathrm{p}<0.05\right.$ vs. $\left.\mathrm{NC}\right)$.

cation influx $\left(\mathrm{Ca}^{2+} / \mathrm{Mg}^{2+}\right)$ and downstream signaling pathways respectively. Moreover, TRPM7 kinase activity can influence its channel function (18).Hence, TRPM7 is a remarkable cation channel with unique kinase activity. TRPM7 channels are highly expressed in CFs and are the major $\mathrm{Ca}^{2+}$-conducting channels in mouse and human CFs (1). $\mathrm{Ca}^{2+}$ signaling is closely implicated in the initiation and development of cardiac fibrosis. Thus, it has been suggested that TRPM7 channels are indispensable for the pro-fibrogenic effect of CFs. Although a variety of studies have explored the relationship between the cation channel TRPM7 and cardiac fibrosis, the present study systematically described for the first time that TRPM7 channels participate in the cardiac fibrosis process via mediating functional changes in CFs. Our primary findings in the present study are as follows. First, we confirmed that the $\mathrm{Ca}^{2+}$ permeable TRPM7 cation channels were expressed in dissociated primary CFs from 1- to 3-day-old SD rat pup hearts. Ang II time-dependently increased TRPM7 mRNA and protein levels and amplified TRPM7 inward-outward rectifying currents in CFs. Secondly, we demonstrated that downregulation of TRPM7 using siRNA interference markedly reduced the Ang II-elicited increase in TRPM7 expression and TRPM7 current enhancement on the CF membrane. Thirdly, we clarified that TRPM7 channels play a pivotal role in Ang II-evoked CF proliferation, differentiation and ECM protein synthesis and silencing of TRPM7 markedly inhibited Ang II-induced CF proliferation, differentiation and ECM protein formation capability. Taken together, our findings suggest that TRPM7 channels act as an essential factor in the Ang II-induced functional change of CFs associated with cardiac fibrosis. The pathological process can be summarized as follows. Under normal physiological conditions, CFs remain quiescent, but are rapidly activated and produce $\mathrm{Ca}^{2+}$ internal flow on the cell membrane upon a variety of pathological damages such as myocardium lesion, pressure overload and oxidative stress. Then they gain contractibility, become proliferative, differentiate into myofibroblasts and generate more pro-fibrogenic factors and ECM proteins ultimately resulting in cardiac fibrosis.

TRPM7 modulates numerous cellular processes in particular the mediation of cell proliferation. As reported previously, TRPM7 was found to modulate the proliferation of malignant human glioma cells (19), mouse cortical astrocytes (20), Ang II-induced rat aortic smooth muscle cells (13) and ox-LDL-induced vascular smooth muscle cells (21). A review by Thodeti et al (3) reported that some TRP channels are associated with CF differentiation. They stated that TRPC3 and TRPM7 predominantly participate in atrial fibroblast differentiation whereas TRPV4 and TRPC6 seemingly are mainly involved in ventricular differentiation (3). However, there appears to be no investigation concentrated on the role of TRPM7 channels in CF proliferation and differentiation. In our in vitro study, we observed that CFs passaged to 3-5 generations exhibited a certain capacity for proliferation (assessed by monitoring CCK-8 and the expression level of Ki-67 and PCNA) and differentiation (assessed by monitoring the expression level of $\alpha$-SMA using immunofluorescence assay and western blot technique) which was markedly increased after Ang II stimulation along with an increase in TRPM7 channel mRNA and protein expression (Figs. 4 and 5). After intervention with Ad-TRPM7-siRNA, the increase in Ang II-elicited proliferation and differentiation in CFs was evidently diminished (Figs. 4 and 5). In addition, using electrophysiological approaches, we found that the TRPM7 currents recorded in the CF cell membrane also manifested a similar trend of change (Fig. 3). These results provided a direct relation between Ang II-induced CF proliferation, differentiation and TRPM7 currents and TRPM7 channels and therefore demonstrated 
that TRPM7 channels mediate Ang II-induced CF proliferation and differentiation by regulating TRPM7 currents.

The ECM protein synthesis levels of CFs under different conditions were previously investigated. Yu and $\mathrm{Xu}$ (22) reported that organ fibrosis including cardiac fibrosis can be deemed as erroneous ECM 'turnover' i.e. imbalance between ECM production (increased) and ECM degradation (reduced). Collagens are the most abundant constructional element of the ECM in the heart, consisting of five types (types I, III, IV, V and VI) found in the myocardium (22). Collagen I and III are the main components of ECM proteins (22). In one of our previous studies, we verified that addition of Ang II to cultured rat CFs notably increased the expression of TRPM7 channels as well as TRPM7 currents and the expression of collagen I and III (4). To further observe the relationship between the ECM protein formation of CFs and TRPM7 channels in the cell membrane, we treated CFs with siRNA targeting TRPM7 in this study. Our results demonstrated that the trend in variation among TRPM7, collagen I and III protein expression levels following treatment with Ang II were consistent with our previous study. The increases in collagen I and III protein induced by Ang II were completely abrogated by TRPM7-siRNA (Fig. 6). This finding together with the above mentioned findings indicate that extracellular Ang II activates TRPM7 channels, increases TRPM7 currents, causes $\mathrm{CF}$ proliferation, differentiation, increased ECM protein synthesis and eventually causes cardiac fibrosis.

In conclusion, the present study provides evidence that TRPM7 channels are indispensable for Ang II-induced proliferation, differentiation and collagen synthesis of CFs via the mediation of TRPM7 currents in the CF cell membrane. More importantly, our findings suggest that by modulating the functional variations in CFs associated with cardiac fibrosis, TRPM7 may act as a promising therapeutic strategy for the treatment of fibrosis-related cardiac diseases. However, our data also demonstrated that TRPM7-siRNA not only inhibited Ang II-elicited $\mathrm{CF}$ proliferation, differentiation and collagen synthesis but also suppressed the proliferation, differentiation and collagen synthesis of CFs in a basal condition. Thus, we have to highlight that in normal conditions, we cannot inhibit the expression of TRPM7 channel and its function in CFs. We can consider inhibiting the expression and function of TRPM7 channels in CFs in patients with cardiac fibrosis-related cardiac diseases for therapeutic use and patients with high risk factors for cardiac fibrosis for prophylactic use. Our future research efforts are as follows: i) to identify the downstream signaling pathways involved in the regulation of the functional changes in CFs by TRPM7 channels; ii) to identify various cardiac-specific TRPM7 inhibitors for future therapeutic use; and iii) to carry out a series of in vivo investigations to identify TRPM7 channels as promising therapeutic targets for fibrosis-related cardiac diseases.

\section{Acknowledgements}

The present study was supported by grants from the National Natural Science Foundation of China(no.81170085), the Natural Science Foundation of Hubei Province (no. 2016CFB162) and the Fundamental Research Funds for the Central Universities (no. 2042016kf0074).

\section{References}

1. Du J, Xie J,Zhang Z, Tsujikawa H, Fusco D, Silverman D, Liang B and Yue L: TRPM7-mediated $\mathrm{Ca}^{2+}$ signals confer fibrogenesis in human atrial fibrillation. Circ Res 106: 992-1003, 2010.

2. Elnakish MT, Kuppusamy P and Khan M: Stem cell transplantation as a therapy for cardiac fibrosis. J Pathol 229: 347-354, 2013.

3. Thodeti CK, Paruchuri S and Meszaros JG: A TRP to cardiac fibroblast differentiation. Channels (Austin) 7: 211-214, 2013.

4. Zhou Y, Yi X, Wang T and Li M: Effects of angiotensin II on transient receptor potential melastatin 7 channel function in cardiac fibroblasts. Exp Ther Med 9: 2008-2012, 2015.

5. Yue L, Xie J and Nattel S: Molecular determinants of cardiac fibroblast electrical function and therapeutic implications for atrial fibrillation. Cardiovasc Res 89: 744-753, 2011.

6. Berridge MJ, Bootman MD and Roderick HL: Calcium signalling: Dynamics, homeostasis and remodelling. Nat Rev Mol Cell Biol 4: 517-529, 2003.

7. González A, López B and Díez J: Fibrosis in hypertensive heart disease: Role of the renin-angiotensin-aldosterone system. Med Clin North Am 88: 83-97, 2004.

8. Olson ER, Shamhart PE, Naugle JE and Meszaros JG: Angiotensin II-induced extracellular signal-regulated kinase 1/2 activation is mediated by protein kinase $\mathrm{C} \delta$ and intracellular calcium in adult rat cardiac fibroblasts. Hypertension 51: 704-711, 2008.

9. Manabe I, Shindo T and Nagai R: Gene expression in fibroblasts and fibrosis: Involvement in cardiac hypertrophy. Circ Res 91: 1103-1113, 2002.

10. Runnels LW, Yue L and Clapham DE: TRP-PLIK, a bifunctional protein with kinase and ion channel activities. Science 291: 1043-1047, 2001.

11. Zhang YH, Sun HY, Chen KH, Du XL, Liu B, Cheng LC, Li X, Jin MW and Li GR: Evidence for functional expression of TRPM7 channels in human atrial myocytes. Basic Res Cardiol 107: 282, 2012.

12. Li M, Jiang J and Yue L: Functional characterization of homoand heteromeric channel kinases TRPM6 and TRPM7. J Gen Physiol 127: 525-537, 2006.

13. Yang M, Zhao T, Lin J, Ju T and Zhang L: Inhibition of TRPM7 attenuates rat aortic smooth muscle cell proliferation induced by angiotensin II: Role of genistein. J Cardiovasc Pharmacol 66: 16-24, 2015.

14. Zheng J: Molecular mechanism of TRP channels. Compr Physiol 3: 221-242, 2013.

15. Montell C, Jones K, Hafen E and Rubin G: Rescue of the Drosophila phototransduction mutation trp by germline transformation. Science 230: 1040-1043, 1985.

16. Nilius B: TRP channels in disease. Biochim Biophys Acta 1772: 805-812, 2007.

17. Visser D, Middelbeek J, van Leeuwen FN and Jalink K: Function and regulation of the channel-kinase TRPM7 in health and disease. Eur J Cell Biol 93: 455-465, 2014.

18. Desai BN, Krapivinsky G, Navarro B, Krapivinsky L, Carter BC, Febvay S, Delling M, Penumaka A, Ramsey IS, Manasian Y, et al: Cleavage of TRPM7 releases the kinase domain from the ion channel and regulates its participation in Fas-induced apoptosis. Dev Cell 22: 1149-1162, 2012.

19. Leng TD, Li MH, Shen JF, Liu ML, Li XB, Sun HW, Branigan D, Zeng Z, Si HF, Li J, et al: Suppression of TRPM7 inhibits proliferation, migration, and invasion of malignant human glioma cells. CNS Neurosci Ther 21: 252-261, 2015.

20. Zeng Z, Leng T, Feng X, Sun H, Inoue K, Zhu L and Xiong ZG: Silencing TRPM7 in mouse cortical astrocytes impairs cell proliferation and migration via ERK and JNK signaling pathways. PLoS One 10: e0119912, 2015.

21. Lin J, Zhou S, Zhao T, Ju T and Zhang L: TRPM7 channel regulates ox-LDL-induced proliferation and migration of vascular smooth muscle cells via MEK-ERK pathways. FEBS Lett 590: 520-532, 2016.

22. Yu LM and Xu Y: Epigenetic regulation in cardiac fibrosis. World J Cardiol 7: 784-791, 2015. 